\title{
A Proposal for Future Modifications on Clinical TNM Staging System of Retinoblastoma Based on the American Joint Committee on Cancer Staging Manual, $7^{\text {th }}$ and $8^{\text {th }}$ Editions
}

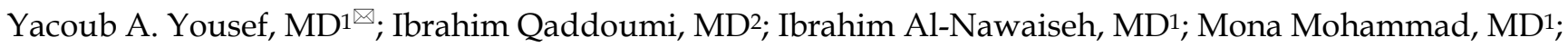
Dalia AlRimawi3 ; Mario Damiano Toro, MD, PhD ${ }^{4,5}$; Sandrine Zweifel, MD ${ }^{4}$; Robert Rejdak, MD, PhD ${ }^{5}$; Rashed Nazzal, MD6; Mustafa Mehyar, MD표 Imad Jaradat, MD7 Iyad Sultan, MD ${ }^{8}$; Maysa Al-Hussaini, $\mathrm{MD}^{9}$

1. Department of Surgery (Ophthalmology), King Hussein Cancer Centre, Amman, Jordan.

2. Department of Oncology, St. Jude Children's Research Hospital, Memphis, TN, USA.

3. Department of Biostatistics, King Hussein Cancer Centre, Amman, Jordan.

4. Department of Ophthalmology, University of Zürich, Zürich, Switzerland.

5. Chair and Department of General and Pediatric Ophthalmology, Medical University of Lublin, Lublin, Poland.

6. Shami Eye Center, Amman, Jordan.

7. Department of Radiation Oncology, King Hussein Cancer Centre, Amman, Jordan.

8. Department of Pediatric Oncology, King Hussein Cancer Centre, Amman, Jordan.

9. Department of Pathology and Laboratory Medicine, King Hussein Cancer Centre, Amman, Jordan.

$\square$ Corresponding authors: Yacoub A. Yousef, MD, Address: Department of Surgery (Ophthalmology), King Hussein Cancer Centre (KHCC), Queen Rania Al-Abdullah Street P.O Box 1269, Amman 11941, Jordan. Tel: +962 787228749 Fax: +962 5300460/1552 Email: yyousef@khcc.jo. Mario D. Toro, MD, PhD, Address: Department of Ophthalmology, University of Zürich, Rämistrasse 100, 8006 Zürich, Switzerland. Tel: +48 815326149 Fax: +48 815326222 Email: toro.mario@email.it.

(1) The author(s). This is an open access article distributed under the terms of the Creative Commons Attribution License (https://creativecommons.org/licenses/by/4.0/). See http://ivyspring.com/terms for full terms and conditions.

Received: 2021.03.29; Accepted: 2021.11.02; Published: 2022.02.07

\begin{abstract}
Importance: The $8^{\text {th }}$ edition of the American Joint Committee on Cancer (AJCC) staging manual incorporated new changes from its 7 th edition for classifying retinoblastoma (RB).

Objective: We assessed the comparative prognostic values of the $7^{\text {th }}$ and $8^{\text {th }}$ editions of the AJCC clinical (cTNM) staging manuals for RB and suggested modifications for future edition accordingly.

Design: A retrospective, observational study.

Setting: King Hussein Cancer Centre.

Participants: A cohort of 478 patients and 565 eyes with RB.

Main Outcomes and Measures: Main outcome measures included demographics; tumor features, AJCC cTNM stage, and eye salvage rates. The prognostic performance of the different staging systems was assessed with the concordance index (C-index) and likelihood ratio $\mathrm{X}^{2}$ tests.

Results: The overall eye salvage rate was $65 \%$. Stage migration occurred for 330 (48\%) eyes with the AJCC Staging Manual, $8^{\text {th }}$ edition. Based on the $7^{\text {th }}$ edition AJCC staging, the eye salvage rate was $94 \%(n=177)$ for T1 tumors (98\% for Tla, 93\%for Tlb, and 90\%for Tlc), $69 \%$ ( $\mathrm{n}=204)$ for T2 tumors (73\% for T2a and 62\%for T2b), and $51 \%(n=40)$ for T3 tumors. Based on the $8^{\text {th }}$ edition AJCC staging, the eye salvage rate was $95 \%(n=139)$ for T1 tumors ( $98 \%$ for Tla and 93\% for T1b), 68\% ( $n=281)$ for T2 tumors (90\%for T2a and 66\%for T2b), and $12 \%$ $(n=1)$ for T3 tumors. With our proposed cTNM modifications, the eye salvage rate was $94 \%(n=177)$ for T1 tumors (98\%for T1a, 93\%for T1b, and $90 \%$ for T1c), $66 \%(n=243)$ for T2 tumors $(73 \%$ for T2a, $62 \%$ for T2b, and $55 \%$ for T2c), and $12 \%(n=1)$ for T3 tumors. As estimated by odds ratios, more advanced cTNM stage (regardless of the cTNM staging system) was significantly associated with an increased chance of treatment failure $(P<.0001)$. The $\mathrm{C}$-index for both the $8^{\text {th }}$ edition and the proposed modifications were approximately equal, and both were higher than that of the $7^{\text {th }}$ edition. However, the proposed modifications had the highest likelihood ratio $X^{2}$ value and the best bootstrap $95 \%$ confidence interval.
\end{abstract}


Conclusions and Relevance: Our proposed modifications on the clinical TNM Staging System for RB harbor more detailed subgroup classification criteria that provides better prognostic value for eye globe salvage than the published similar (but not identical) AJCC Staging Manual, 7th and $8^{\text {th }}$ editions, furthermore these modifications may resolve the discrepancies in the previously published different classification systems for RB.

Key words: Retinoblastoma; Globe salvage; Staging system; Prognosis; American Joint Committee on Cancer

\section{Introduction}

Retinoblastoma (RB) is the most common primary ocular malignant neoplasm of childhood. The prognosis for long-term survival is excellent in developed countries, where most tumors are intraocular at presentation $[1,2,3]$. The likelihood of globe salvage depends on many tumor features, such as size, presence of subretinal fluid, vitreous seeds, and subretinal seeding $[4,5]$.

Eye salvage rates are as high as $70 \%$ to $100 \%$ for smaller tumors (group A-C) but are as low as $23 \%$ for advanced tumors (group D or E) [6,7,8]. Moreover, treatment burden increases with tumor size and severity. Although group A tumors can be treated with such focal therapies as laser or cryotherapy, large tumors or tumors with seeding require more invasive therapies, such as systemic chemotherapy, intraarterial chemotherapy (IAC), intravitreal chemotherapy (IViC), or radioactive plaque brachytherapy $[9,10,11,12,13]$.

The American Joint Committee on Cancer (AJCC) tumor/node/metastasis (TNM) staging system, which is a universal staging system for all cancers, included both intraocular and extraocular RB tumors in the $7^{\text {th }}$ edition of the AJCC Staging Manual (AJCC-7) in 2009 [11]. This was updated by 18 RB specialist centers from 13 countries in the $8^{\text {th }}$ edition of the AJCC Staging Manual (AJCC-8) in 2017 [14] and is suggested to most accurately predict eye salvage, metastasis, and death. AJCC-8 incorporates intraocular (cT1-cT3) and extraocular (cT4) tumors and includes heritability, making RB the first cancer to consider heritability in its staging [15].

The AJCC-8 merged all eyes with vitreous and sub retinal seeds (regardless severity, location, and extent) in one category (cT2b), while we believe it is better for these eyes to be divided into further subgroups to give better prognostic power for this staging system. Furthermore, the AJCC staging team analyzed a heterogonous group of patients treated by different teams in different countries across the world. This heterogeneity empowered the AJCC-8, even though, we believe it should be helpful to validate this staging system based on a homogenous group of patients treated by the same team in one specialized center as well. Herein, we evaluated the discriminative ability of both the AJCC-7 and AJCC-8 clinical TNM (cTNM) staging systems for intraocular
$\mathrm{RB}$ tumors and the effect of stage migration from AJCC-7 to AJCC-8 on the prognosis of globe salvage by performing a retrospective analysis of patients with RB who were treated at King Hussein Cancer Center (KHCC) in Amman, Jordan from 2003 to 2019. On the basis of our findings, we put forward suggestions for modifications on AJCC-8 for grouping for RB to be evaluated by different single center and multicenter studies and thereafter may be considered for future TNM staging edition to better predict the likelihood of globe salvage.

\section{Methods}

This is a retrospective cohort study of 478 patients (697 eyes) who had clinically diagnosed RB and were treated at KHCC from 2003 to 2019. The KHCC Institutional Review Board approved this study. Selection required access to patient medical records and RetCam images. The data collected included patient demographics, treatment modalities, eye salvage, metastasis, mortality rates, and tumor features and stage at diagnosis. All tumors were restaged according to AJCC-7 and AJCC-8 criteria (eTables 1 and 2 in Supplement 1) [14,15].

\section{Inclusion and Exclusion Criteria}

The inclusion criteria for this study comprised eyes with a clinical diagnosis of intraocular RB that received conservative treatment to avoid enucleation and/or external beam radiation therapy (EBRT). The exclusion criteria consisted of cases without follow-up records, eyes with extraocular tumor invasion, and tumors treated with primary enucleation or EBRT. We defined eye salvage as the absence of tumor activity for at least 6 months after the last active treatment.

\section{Tumor Features, Definitions, TNM Clinical Staging, and Treatment Modalities}

We reviewed RetCam images and clinical drawings for all eyes at the time of diagnosis and documented each tumor feature. We then restaged the tumors according to AJCC-7 and AJCC-8 TNM criteria for RB (eTables 1 and 2 in Supplement 1) $[14,15]$.

We defined intraretinal tumors as those involving the retina, without subretinal seeding or vitreous seeds. Large tumors were defined as those 
filling more two-thirds of the eye globe, as detected clinically, in B-scan ultrasounds, and/or in magnetic resonance images. We classified tumor seeds as subretinal or vitreous according to their location. The severity of the seeds was classified as focal vitreous and/or subretinal if fine aggregates of tumor cells were present without large clumps or "snowballs" or as massive vitreous and/or subretinal seeding if diffuse clumps or snowballs of tumor cells were present.

The standard treatment for RB consisted of a systemic chemotherapy regimen of carboplatin, vincristine, and etoposide combined with focal consolidation therapy. Each chemotherapy cycle was repeated every 3 to 4 weeks for a total of 6 to 8 cycles, according to patient condition and tumor status. Ocular oncology follow-up was provided with examination under anesthesia before each cycle of chemotherapy and every 3 to 4 weeks thereafter. Fundus photos were taken with a RetCam II instrument (Clarity Medical System, Pleasanton, CA, USA). Focal therapy was applied when needed as transpupillary thermotherapy and/or triple freeze thaw cryotherapy (MIRA CR 4000), starting after the second cycle of systemic chemotherapy. IAC, IViC, subtenon chemotherapy, and $\mathrm{I}^{125}$ radioactive plaque brachytherapy were used as second-line treatment options for tumor recurrence or for residual tumor activity. For this study, we defined treatment failure as the need for EBRT or enucleation.

\section{A Proposed Modifications on cTNM Classification System}

We suggested new modifications on the CTNM classification system to be considered for future edition of the AJCC Staging Manual for RB (Table 1). Based on our suggested modifications, tumors were divided into $4 \mathrm{cT}$ stages according to their clinical features. The cT1 and CT2 stages comprised potentially salvageable eyes with intraocular RB that had nearly no risk for metastasis. The cT3 stage comprised eyes with intraocular RB that were generally unsalvageable and were more likely to harbor high-risk pathologic features $[15,16]$ and therefore had a higher risk of metastasis than CT1 and cT2 tumors. The cT4 stage comprised eyes with extraocular RB. We further divided the CT1 and CT2 stages into 3 homogenous subgroups according to their likelihood of eye salvage. The higher-stage groups and subgroups were expected to have a higher likelihood of treatment failure.

Furthermore, we divided cT3 group into 2 subgroups (Table 1). The rationale behind this modification was that cT3a in our suggested module are features that are secondary to ocular ischemia due to huge tumor size, severe RD, and/or secondary to increased IOP. This category of eyes are expected to have low potential for eye and vision salvage, but relatively low impact on metastatic chance as they do not invade vital structures and will not show high risk pathological features compare to the next subgroup. $[16,17]$ In the other hand, cT3b in our suggested modifications are eyes with tumor invasion into vital structures that may cause metastasis (as ciliary body and anterior chamber), and therefore trial of eye salvage in this case will have more risk for metastasis than CT3a and may increase the mortality rate.

Table 1. Proposal for Modifications on the Definition of cT in the AJCC cTNM Staging Classification System for Retinoblastoma ${ }^{a}$

\footnotetext{
Stage Tumor Characteristics

cTX Unknown evidence of intraocular tumor

cT0 No evidence of intraocular tumor

cT1 Intraretinal tumor(s) occupying $<2 / 3$ globe, with subretinal fluid $\leq$ quadrant of the globe;

No vitreous or subretinal seeding is allowed

cT1a Tumors $\leq 3 \mathrm{~mm}$ and further than $1.5 \mathrm{~mm}$ from the disc and fovea

cT1b Tumors $>3 \mathrm{~mm}$ or closer than $1.5 \mathrm{~mm}$ to the disc and fovea; No retinal detachment or subretinal fluid beyond $5 \mathrm{~mm}$ from the base of the tumor

cT1c Subretinal fluid $>5 \mathrm{~mm}$ from the base of any tumor and up to quadrant of the globe

cT2 Intraocular tumor(s) with retinal detachment, vitreous seeding, or subretinal seeding

cT2a Tumors with focal vitreous seeding and/or subretinal seeding Retinal detachment $>$ quadrant of the globe but $<$ total

cT2b Tumors with massive vitreous seeding and/or subretinal seeding and/or total exudative retinal detachment

CT2c Large intraocular tumors occupying more than $2 / 3$ of the eye globe

cT3 Advanced intraocular tumor(s)

CT3a Raised intraocular pressure with neovascularization and/or buphthalmos; Hyphema and/or massive vitreous hemorrhage

cT3b Tumor invasion of the pars plana, ciliary body, lens, zonules, iris or anterior chamber; Phthisis or pre-phthisis bulbi or aseptic orbital cellulitis

cT4 Extraocular tumor(s) involving the orbit, including the optic nerve

cT4a Radiological evidence of retrobulbar optic nerve involvement or thickening of the optic nerve or involvement of the orbital tissues

cT4b Extraocular tumor clinically evident with proptosis and orbital mass

Abbreviations: AJCC, American Joint Committee on Cancer; cTNM, clinical tumor/node/metastasis.

antraretinal tumors were defined as tumors involving the retina without subretinal seeding or vitreous seeds. Focal vitreous and/or subretinal seeding were defined as fine aggregates of tumor cells, and massive vitreous and/or subretinal seeding were defined as diffuse clumps or "snowballs" of tumor cells are present. Large tumors were defined as tumors filling more $2 / 3$ of the eye globe, as detected clinically, in B-scan ultrasounds, and/or with magnetic resonance imaging.
}

\section{Statistical Methods}

The primary endpoint of this study was globe salvage. The multivariate Cox proportional hazard model was used to evaluate hazard ratios and 95\% confidence intervals for the known prognostic power of all of the TNM stages. The discriminatory ability of the staging systems was measured by the concordance index (C-index). [17] The prognostic homogeneity of the staging systems was assessed with likelihood ratio $\chi^{2}$ tests. Higher C-index and likelihood ratio $\chi^{2}$ values were indicative of improved performance of the staging systems. We used SAS software v9.4 (SAS Institute Inc, Cary, NC) for 
statistical analyses. The odds ratio estimates for different stages were measured with a logistic regression model, and the point effect was set as group A or CT1a for the probability of treatment failure. $P$ values were measured with Fisher exact tests, and values of 0.05 or less were considered significant.

\section{Results}

\section{Patients Demographics and Management Outcomes}

We analyzed clinical data from 478 patients: 249 (52\%) were boys, and 335 (70\%) had bilateral disease. We investigated 697 eyes with RB tumors: 6 had extraocular RB, 126 were treated with primary enucleation, and 565 were managed by conservative therapy targeting globe salvage. A family history of $\mathrm{RB}$ was present in $6(4 \%)$ unilateral cases and in 66 $(20 \%)$ bilateral cases. The median age at diagnosis was 6 and 28 months for patients with bilateral and unilateral RB, respectively.

Of 565 eyes that received conservative therapy, $421(75 \%)$ eyes were salvaged, $369(65 \%)$ were treated with chemoreduction alone, and $52(9 \%)$ mandated additional therapy, such as IAC (15 eyes), IViC (21 eyes), subtenon chemotherapy (24 eyes), or $\mathrm{I}^{125}$ radioactive plaque therapy (13 eyes). Treatment failure occurred for 144 (25\%) eyes: 130 eyes were enucleated (including 16 eyes that also received EBRT), and 14 eyes were preserved with EBRT. After a 120-month median follow-up period, 24 (5\%) patients died of second neoplasms $(\mathrm{n}=3)$ or metastases $(\mathrm{n}=21)$.

\section{Tumor Features according to the AJCC-7 and AJCC-8 cTNM Staging Systems}

For the 565 eyes that received conservative therapy, tumors in 488 eyes were smaller than two-thirds of the globe, and 77 were larger than two-thirds of the globe. Tumors were intraretinal in 147 eyes and extraretinal in 418 eyes. Tumor seeding was present in 368 eyes: 151 eyes had subretinal seeds, 119 eyes had vitreous seeds, and 98 eyes had both types of seeds. We detected focal seeds in 209 eyes and massive seeds in 159 eyes (Table 2).

According to AJCC-7, 189 (27\%) eyes were T1 ( $6 \%$ were $\mathrm{T} 1 \mathrm{a}, 15 \%$ were $\mathrm{T} 1 \mathrm{~b}$, and $6 \%$ were $\mathrm{T} 1 \mathrm{c}), 345$ $(49 \%)$ were T2 (29\% were T2a and 20\% were T2b), 157 $(23 \%)$ were $\mathrm{T} 3(12 \%$ were $\mathrm{T} 3 \mathrm{a}$ and $11 \%$ were $\mathrm{T} 2 \mathrm{~b})$, and 6 were T4. According to AJCC-8, 147 (21\%) eyes were $\mathrm{T} 1$ (6\% were $\mathrm{T} 1 \mathrm{a}$ and $15 \%$ were $\mathrm{T} 1 \mathrm{~b}), 471(68 \%)$ were T2 (6\% were T2a and $62 \%$ were T2b), 73 (11\%) were T3, and 6 were T4 (Table 3).

\section{Stage Migration}

Among the 691 eyes with intraocular RB, 361 $(52 \%)$ had the same stage in both the AJCC-7 and AJCC-8 classification systems, including all eyes in stages cT1a and cT1b and some eyes in stages cT2a, cT2b, and cT3 (Table 4). Stage migration occurred for $330(48 \%)$ eyes, including $246(36 \%)$ eyes that were upstaged (ie, the stage in AJCC- 8 was higher than that in the AJCC-7) and $84(12 \%)$ that were downstaged (ie, the stage in AJCC-8 was lower than that in AJCC-7). Specifically, $42(6 \%)$ eyes were upstaged from cT1c to cT2b, $204(30 \%)$ upstaged from cT2a to cT2b, and $84(12 \%)$ eyes were downstaged from cT3 to $\mathrm{cT} 2 \mathrm{~b}$.

Table 2. Association of Tumor Features and Globe Salvage

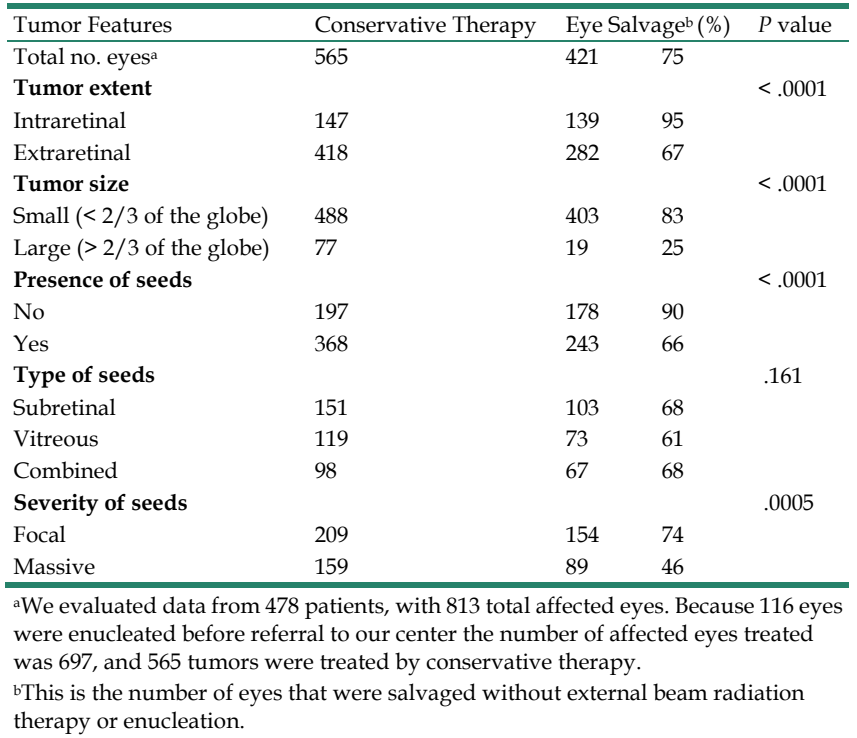

\section{Correlation between Tumor Features and Eye Salvage}

We found $95 \%(n=139 / 147)$ of eyes with intraretinal tumors were salvaged, whereas $67 \%(\mathrm{n}=$ $282 / 418$ ) of eyes with extraretinal tumors were salvaged. Therefore, tumor extent was a significant risk factor for treatment failure $(P<.0001)$. We found $83 \%$ ( $n=403 / 488)$ of eyes with small tumors were salvaged, whereas only $25 \%(n=19 / 77)$ of eyes with large tumors were salvaged. Therefore, tumor size was also significant risk factor for treatment failure $(P$ $<.0001)$. The presence of tumor seeding was significant as a risk factor for failure of local control $(P$ $<.0001)$, as $90 \%(\mathrm{n}=178 / 197)$ of eyes without seeding and $66 \%(n=243 / 368)$ of eyes with seeding were salvaged. In addition, tumors with massive seeds were more likely to fail treatment than those with focal seeds $(P=.0005)$, as $74 \%(n=154 / 209)$ eyes with focal seeds and only $46 \%(n=89 / 159)$ with massive 
seeds were salvaged. Notably, the type of seeding did not significantly affect the salvage rate $(P=.161)$, although eyes with vitreous seeds were marginally less likely to be salvaged $(61 \%)$ than were those with subretinal seeds (68\%) (Table 2$)$.

Table 3. Tumor cTNM Staging and Management Outcomes

\begin{tabular}{|c|c|c|c|c|}
\hline & Number (\%) & $\begin{array}{l}\text { Primary } \\
\text { Enucleation (\%) }\end{array}$ & $\begin{array}{l}\text { Amended } \\
\text { Treatment }\end{array}$ & $\begin{array}{l}\text { Overall Eye Salvage for } \\
\text { Amended Treatment }\end{array}$ \\
\hline $\begin{array}{l}\text { Total no. } \\
\text { eyes }^{\mathrm{a}}\end{array}$ & 697 & $132(19)$ & 565 & $421(75)$ \\
\hline \multicolumn{5}{|l|}{ AJCC-7 } \\
\hline T1 & $189(27)$ & $0(0)$ & 189 & $177(94)$ \\
\hline T1a & $41(6)$ & $0(0)$ & 41 & $40(98)$ \\
\hline $\mathrm{T} 1 \mathrm{~b}$ & $106(15)$ & $0(0)$ & 106 & $99(93)$ \\
\hline T1c & $42(6)$ & $0(0)$ & 42 & $38(90)$ \\
\hline T2 & $345(49)$ & $48(14)$ & 297 & $204(69)$ \\
\hline T2a & $204(29)$ & $26(13)$ & 176 & $129(73)$ \\
\hline $\mathrm{T} 2 \mathrm{~b}$ & $141(20)$ & $22(16)$ & 121 & $75(62)$ \\
\hline T3 & $157(23)$ & $78(50)$ & 79 & $40(51)$ \\
\hline T3a & $84(12)$ & $13(16)$ & 71 & $39(55)$ \\
\hline $\mathrm{T} 3 \mathrm{~b}$ & $73(11)$ & $65(89)$ & 8 & $1(12)$ \\
\hline $\mathrm{T} 4^{\mathrm{b}}$ & $6(<1)$ & $6(100)$ & 0 & $0(0)$ \\
\hline \multicolumn{5}{|l|}{ AJCC-8 } \\
\hline T1 & $147(21)$ & $0(0)$ & 147 & 139 (95) \\
\hline T1a & $41(6)$ & $0(0)$ & 41 & $40(98)$ \\
\hline $\mathrm{T} 1 \mathrm{~b}$ & $106(15)$ & $0(0)$ & 106 & $99(93)$ \\
\hline $\mathrm{T} 2$ & $471(68)$ & $61(13)$ & 410 & $281(68)$ \\
\hline $\mathrm{T} 2 \mathrm{a}$ & $42(6)$ & $0(0)$ & 42 & $38(90)$ \\
\hline $\mathrm{T} 2 \mathrm{~b}$ & $429(62)$ & $61(14)$ & 368 & $243(66)$ \\
\hline T3 & $73(11)$ & $65(89)$ & 8 & $1(12)$ \\
\hline T3a & $23(3)$ & $21(91)$ & 2 & $0(0)$ \\
\hline $\mathrm{T} 3 \mathrm{~b}$ & $11(2)$ & $10(91)$ & 1 & $0(0)$ \\
\hline $\mathrm{T} 3 \mathrm{c}$ & $28(4)$ & $24(86)$ & 4 & $1(25)$ \\
\hline T3d & $7(1)$ & $6(86)$ & 1 & $0(0)$ \\
\hline \multirow[t]{2}{*}{ T3e } & $4(1)$ & $4(100)$ & 0 & $0(0)$ \\
\hline & $6(<1)$ & $6(100)$ & 0 & $0(0)$ \\
\hline T4 & $6(<1)$ & $6(100)$ & 0 & $0(0)$ \\
\hline T4a & $5(<1)$ & $5(100)$ & 0 & \\
\hline $\mathrm{T} 4 \mathrm{~b}$ & $1(<1)$ & $1(100)$ & 0 & \\
\hline \multicolumn{5}{|c|}{ Proposed Modified cTNM Staging System } \\
\hline T1 & $189(27)$ & $0(0)$ & 189 & $177(94)$ \\
\hline T1a & $41(6)$ & $0(0)$ & 41 & $40(98)$ \\
\hline $\mathrm{T} 1 \mathrm{~b}$ & $106(15)$ & $0(0)$ & 106 & $99(93)$ \\
\hline T1c & $42(6)$ & $0(0)$ & 42 & $38(90)$ \\
\hline T2 & $429(62)$ & $61(14)$ & 368 & $243(66)$ \\
\hline $\mathrm{T} 2 \mathrm{a}$ & $204(29)$ & $26(13)$ & 176 & $129(73)$ \\
\hline $\mathrm{T} 2 \mathrm{~b}$ & $141(20)$ & $22(16)$ & 121 & 75 (62) \\
\hline $\mathrm{T} 2 \mathrm{c}$ & $84(12)$ & $13(16)$ & 71 & $39(55)$ \\
\hline T3 & $73(11)$ & $65(89)$ & 8 & $1(12)$ \\
\hline $\mathrm{T} 2 \mathrm{a}$ & $35(5)$ & $30(86)$ & 5 & $1(20)$ \\
\hline $\mathrm{T} 2 \mathrm{~b}$ & $38(6)$ & $35(92)$ & 3 & $0(0)$ \\
\hline T4 & $6(<1)$ & $6(100)$ & 0 & $0(0)$ \\
\hline Metastasis $^{c}$ & $22(4)$ & & & \\
\hline $\begin{array}{l}\text { Secondary } \\
\text { malignancy }\end{array}$ & $4(1)$ & & & \\
\hline Mortality $^{\mathrm{d}}$ & $24(5)$ & & & \\
\hline
\end{tabular}

Abbreviations: AJCC-7, American Joint Committee on Cancer Staging Manual, 7th edition; AJCC-8, American Joint Committee on Cancer Staging Manual, 8th edition; cTNM, clinical tumor/node/metastasis.

a We evaluated data from 478 patients, with 813 total affected eyes. Because 116 eyes were enucleated before referral to our center, the number of affected eyes treated was 697.

b Of 126 eyes treated with primary enucleation, 6 had extraocular disease treated by enucleation after neoadjuvant chemotherapy.

c This included all patients with metastasis including those who had metastasis at time of diagnosis and those who developed metastasis during therapy and follow up at our center. All but one died because of metastasis.

d Twenty four patients died at the last date of follow up; 21 with metastasis and 3 with second malignancy.
Table 4. Distribution of Tumors according to the AJCC cTNM Staging Manual, $7^{\text {th }}$ and $8^{\text {th }}$ Editions

\begin{tabular}{lllllll}
\hline & \multicolumn{3}{l}{ AJCC-8 cTNM Stage } & Sum \\
\cline { 2 - 5 } & $1 \mathrm{a}$ & $1 \mathrm{~b}$ & $2 \mathrm{a}$ & $2 \mathrm{~b}$ & 3 & \\
\hline AJCC-7 cTNM Stage & & & & & & \\
$1 \mathrm{a}$ & 41 & 0 & 0 & 0 & 0 & 41 \\
$1 \mathrm{~b}$ & 0 & 106 & 0 & 0 & 0 & 106 \\
$1 \mathrm{c}$ & 0 & 0 & 42 & 0 & 0 & 42 \\
$2 \mathrm{a}$ & 0 & 0 & 0 & 204 & 0 & 204 \\
$2 \mathrm{~b}$ & 0 & 0 & 0 & 141 & 0 & 141 \\
3 & 0 & 0 & 0 & 84 & 73 & 157 \\
Sum & 41 & 106 & 42 & 429 & 73 & 691 \\
\hline
\end{tabular}

Abbreviations: Abbreviations: AJCC-7, American Joint Committee on Cancer Staging Manual, 7th edition; AJCC-8, American Joint Committee on Cancer Staging Manual, $8^{\text {th }}$ edition; cTNM, clinical tumor/node/metastasis.

\section{Correlation between AJCC-7 and Eye Salvage}

More advanced cTNM-staged tumors had a significantly higher likelihood of treatment failure than did those with lower cTNM stages $(P<0.0001)$. The eye salvage rate was $94 \%(\mathrm{n}=177 / 189)$ for $\mathrm{T} 1$ tumors (98\% for T1a, 93\% for T1b, and $90 \%$ for $\mathrm{T} 1 \mathrm{c})$, $69 \%(\mathrm{n}=204 / 297)$ for T2 (73\% for T2a and $62 \%$ for $\mathrm{T} 2 \mathrm{~b})$, and $51 \%(\mathrm{n}=40 / 79)$ for T3 $(55 \%$ for $\mathrm{T} 3 \mathrm{a}$ and $12 \%$ for T2b). A logistic regression model demonstrated that the treatments for $\mathrm{T} 2$ and $\mathrm{T} 3$ tumors were 6.7 and 14 times more likely to fail than were those for $\mathrm{T} 1$ tumors, respectively, and the treatments for T1b, T1c, T2a, and T2b tumors were 2.8, $4.2,14.6$, and 24.5 times more likely to fail than were those for T1a tumors, respectively (Table 5).

\section{Correlation between AJCC-8 and Eye Salvage}

More advanced cTNM-staged tumors had a significantly higher likelihood of treatment failure than did those with lower cTNM stages $(P<.0001)$. The eye salvage rate was $95 \%(\mathrm{n}=139 / 147)$ for $\mathrm{T} 1$ tumors $(98 \%$ for $\mathrm{T} 1 \mathrm{a}$ and $93 \%$ for $\mathrm{T} 1 \mathrm{~b}), 68 \%$ (n = $281 / 410)$ for T2 (90\% for T2a and $66 \%$ for T2b), and $12 \%(\mathrm{n}=1 / 8)$ for T3. A logistic regression model showed that the treatments for T2 and T3 tumors were 7.98 and 121.6 times more likely to fail than were those for $\mathrm{T} 1$ tumors, respectively, and the treatments for $\mathrm{T} 1 \mathrm{~b}, \mathrm{~T} 2 \mathrm{a}$, and $\mathrm{T} 2 \mathrm{~b}$ tumors were $2.8,4.2$, and 20.6 times more likely to fail than were those for T1a tumors, respectively (Table 5).

\section{A Proposal for Modifications on the cTNM Staging System}

Because $95 \%$ of eyes with intraretinal tumors and $67 \%$ of those with extraretinal tumors were salvaged $(P<.0001)$, we consolidated intraretinal tumors in the same stage (cT1), which we subdivided into 3 subgroups according to tumor size and severity. Because the severity of tumor seeds in extraretinal tumors affected prognosis, we assigned tumors with massive seeds as more advanced (cT2b) than tumors with focal seeds (cT2a). Only $25 \%$ of eyes with large 
tumors were salvaged, which was worse than eyes containing massive seeds; therefore, we assigned large tumors as CT2c. Because the type of seeding did not affect the eye salvage rate $(P=.161)$, we did not consider seed type as a factor for staging. We assigned potentially unsalvageable intraocular group E tumors harboring high-risk pathologic features as stage T3 $[15,16]$ and those with extraocular invasion as stage $\mathrm{T} 4$.

Table 5. Prognostic Performance of AJCC-7, AJCC-8, and the Proposed Modifications on cTNM Staging Systems

\begin{tabular}{|c|c|c|c|c|c|}
\hline & \multicolumn{4}{|c|}{ Concordance Indices } & \multirow{2}{*}{$\begin{array}{l}\text { Likelihood Ratio } \\
X^{2}\end{array}$} \\
\hline & \multicolumn{2}{|l|}{ C-index } & \multicolumn{2}{|c|}{ Bootstrap 95\% CI } & \\
\hline AJCC-7 & \multicolumn{2}{|l|}{0.6927} & \multicolumn{2}{|c|}{$0.6391-0.7464$} & $(29.51)<.0001$ \\
\hline AJCC-8 & \multicolumn{2}{|l|}{0.7454} & \multicolumn{2}{|c|}{$0.6203-0.8706$} & $(18.49)<.0001$ \\
\hline \multirow{3}{*}{$\begin{array}{l}\text { Propose } \\
\text { d System }\end{array}$} & \multicolumn{2}{|l|}{0.7249} & \multicolumn{2}{|c|}{$0.6714-0.7785$} & $(42.71)<.0001$ \\
\hline & & & \multicolumn{2}{|c|}{ Odds Ratio Estimates } & \\
\hline & $\begin{array}{l}\text { Eye Salvage } \\
(\%)\end{array}$ & Effect & $P$ Value & $\begin{array}{l}\text { Point } \\
\text { Estimate }\end{array}$ & $\begin{array}{l}\text { 95\% Wald } \\
\text { Confidence } \\
\text { Limits }\end{array}$ \\
\hline \multicolumn{6}{|l|}{ AJCC-7 } \\
\hline T1 & $177(94)$ & - & & & \\
\hline T1a & $40(98)$ & - & & & \\
\hline $\mathrm{T} 1 \mathrm{~b}$ & $99(93)$ & T1b vs T1a & .3381 & 2.828 & $0.337-23.732$ \\
\hline T1c & $38(90)$ & T1c vs T1a & .2076 & 4.210 & $0.450-39.384$ \\
\hline T2 & $204(69)$ & $\mathrm{T} 2$ vs $\mathrm{T} 1$ & $<.0001$ & 6.724 & $3.567-12.676$ \\
\hline $\mathrm{T} 2 \mathrm{a}$ & $129(73)$ & T2a vs T1a & .0091 & 14.573 & $1.948-109.002$ \\
\hline $\mathrm{T} 2 \mathrm{~b}$ & $75(62)$ & T2b vs T1a & .0019 & 24.533 & $3.261-184.554$ \\
\hline T3 & $40(51)$ & T3 vs T1 & $<.0001$ & 14.381 & $6.914-29.912$ \\
\hline T3a & $39(55)$ & T3a vs T1a & .0008 & 32.819 & $4.273-252.050$ \\
\hline $\mathrm{T} 3 \mathrm{~b}$ & $1(12)$ & T3bvs T1a & .0001 & 279.991 & 15.627 - >999.999 \\
\hline \multicolumn{6}{|l|}{ AJCC-8 } \\
\hline T1 & $139(95)$ & - & & & \\
\hline T1a & $40(98)$ & - & & & \\
\hline $\mathrm{T} 1 \mathrm{~b}$ & $99(93)$ & T1b vs T1a & .3381 & 2.828 & $0.337-23.732$ \\
\hline T2 & $281(68)$ & $\mathrm{T} 2$ vs $\mathrm{T} 1$ & $<.0001$ & 7.976 & $3.796-16.758$ \\
\hline $\mathrm{T} 2 \mathrm{a}$ & $38(90)$ & T2a vs T1a & .2076 & 4.21 & $0.45-39.384$ \\
\hline $\mathrm{T} 2 \mathrm{~b}$ & $243(66)$ & T2b vs T1a & .003 & 20.575 & $2.796-151.422$ \\
\hline T3 & $1(12)$ & T3 vs T1 & $<.0001$ & 121.615 & 13.299 - >999.999 \\
\hline \multicolumn{6}{|c|}{ Proposed Modified cTNM Staging System } \\
\hline T1 & 177 (94) & - & & & \\
\hline T1a & $40(98)$ & - & & & \\
\hline $\mathrm{T} 1 \mathrm{~b}$ & $99(93)$ & T1b vs T1a & .3381 & 2.828 & $0.337-23.732$ \\
\hline
\end{tabular}

\begin{tabular}{llllll}
\hline \multicolumn{5}{c}{ Concordance Indices } & \multicolumn{2}{l}{\begin{tabular}{l} 
Likelihood Ratio \\
\cline { 2 - 5 }
\end{tabular}} & C-index & & \multicolumn{2}{l}{ Bootstrap 95\% CI } & $\chi^{2}$ \\
\hline T1c & $38(90)$ & T1c vs T1a & .2076 & 4.210 & $0.450-39.384$ \\
T2 & $243(66)$ & T2 vs T1 & $<.0001$ & 6.127 & $3.275-11.461$ \\
T2a & $129(73)$ & T2a vs T1a & .0091 & 14.573 & $1.948-109.002$ \\
T2b & $75(62)$ & T2b vs T1a & .0019 & 24.533 & $3.261-184.554$ \\
T2c & $39(55)$ & T2c vs T1a & .0008 & 32.819 & $4.273-252.050$ \\
T3 & $1(12)$ & T3 vs T1 & $<.0001$ & 103.247 & $11.726-909.088$ \\
\hline
\end{tabular}

Abbreviations: AJCC-7, American Joint Committee on Cancer Staging Manual, 7th edition; AJCC-8, American Joint Committee on Cancer Staging Manual, 8th edition; cTNM, clinical tumor/node/metastasis.

According to our proposed cTNM staging system, $189(27 \%)$ eyes were $\mathrm{T} 1$ (6\% were $\mathrm{T} 1 \mathrm{a}, 15 \%$ were $\mathrm{T} 1 \mathrm{~b}$, and $6 \% \mathrm{~T} 1 \mathrm{c}), 429(62 \%)$ were $\mathrm{T} 2(29 \%$ were $\mathrm{T} 2 \mathrm{a}, 20 \%$ were $\mathrm{T} 2 \mathrm{~b}$, and $12 \% \mathrm{~T} 2 \mathrm{c}), 73(11 \%)$ were $\mathrm{T} 3$ $(5 \%$ were $\mathrm{T} 3 \mathrm{a}$, and $6 \%$ were $\mathrm{T} 3 \mathrm{~b})$, and 6 were $\mathrm{T} 4$ (Table 3). Advanced cTNM-staged tumors had a significantly higher likelihood of treatment failure $(P$ $<.0001)$. The eye salvage rate was $94 \%(\mathrm{n}=177 / 189)$ for $\mathrm{T} 1$ tumors $(98 \%$ for $\mathrm{T} 1 \mathrm{a}, 93 \%$ for $\mathrm{T} 1 \mathrm{~b}$, and $90 \%$ for T1c), $66 \%(n=243 / 368)$ for T2 (73\% for T2a, $62 \%$ for $\mathrm{T} 2 \mathrm{~b}$, and $55 \%$ for $\mathrm{T} 2 \mathrm{c})$, and $12 \%(\mathrm{n}=1 / 8)$ for T3 $(17 \%$ for $\mathrm{T} 3 \mathrm{a}$ and $0 \%$ for $\mathrm{T} 2 \mathrm{~b})$. Logistic regression demonstrated that the treatments for $\mathrm{T} 2$ and $\mathrm{T} 3$ tumors were 6.1 and 103 times more likely to fail than were those for $\mathrm{T} 1$ tumors, respectively, and the treatments for $\mathrm{T} 1 \mathrm{~b}, \mathrm{~T} 1 \mathrm{c}, \mathrm{T} 2 \mathrm{a}, \mathrm{T} 2 \mathrm{~b}$, and $\mathrm{T} 2 \mathrm{c}$ tumors were $2.8,4.2,14.6,24.5$, and 32.8 times more likely to fail than were those for T1a tumors, respectively (Table 5).

The 3 TNM staging systems (AJCC-7, AJCC-8, and the modified staging system) we investigated were all generally able to predict the likelihood of eye salvage (Figure 1 and Table 5). We compared the performance of AJCC-7, AJCC-8, and the modified staging system by calculating the C-index and likelihood ratio $X^{2}$ values (Table 5). The modified staging system had the highest likelihood ratio $X^{2}$

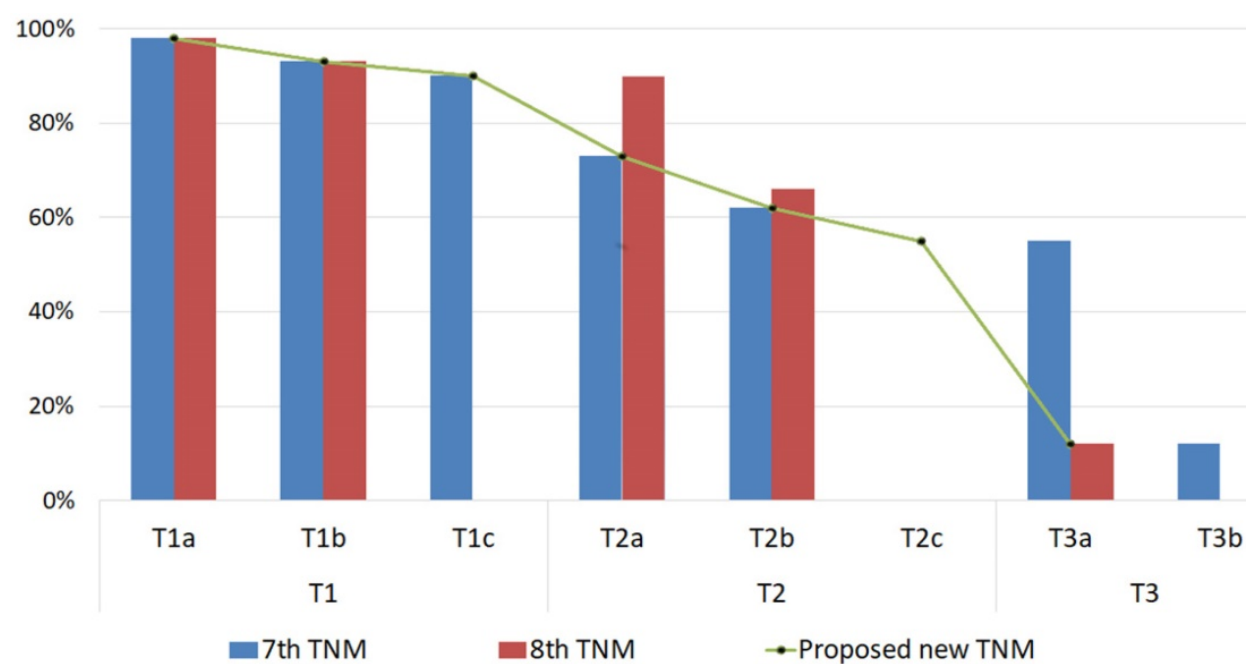

Figure 1. Globe Salvage Rates of Eyes with Intraocular Retinoblastoma. The globe salvage rates are shown for tumors classified with the American Joint Committee on Cancer Staging Manual, $7^{\text {th }}$ edition and $8^{\text {th }}$ editions and our proposed cTNM staging system modifications. 
value. The C-index values for both AJCC-8 and the proposed system were approximately equal (both were higher than that of AJCC-7), but the confidence interval for the proposed staging system was (0.67-0.77) which is better than the confidence interval for AJCC-8 (0.62-0.87), indicating that our proposed staging system has better prognostic capability than AJCC-8.

\section{Correlation between AJCC-7, AJCC-8, and the proposed modifications and the Rate of Metastasis}

Twenty-one patients developed metastasis; no patient with the worst eye stage $\mathrm{T} 1$ developed metastasis in any of the three TNM staging systems. Four patients had extraocular RB and were stage T4 in the three systems, and eight patients had the worst eye staged as T3b based on AJCC-7, and were staged T3 based on both AJCC-8 and the proposed modifications (Table 6). Because of the low number of patients with metastasis in each subgroup, no statistical correlation could be done (Figure 2).

\section{Discussion}

Accurate staging is essential to guide treatment and predict prognosis $[18,19]$. When we evaluated the discriminative ability of AJCC-7 and AJCC-8 to predict the likelihood of globe salvage, we found that higher cT stage in both editions was associated with an increased risk of treatment failure. However, our proposed staging system exhibited the best performance of the 3 , indicating that our proposed modifications are better for predicting globe salvage.

Recently, the predictive value of the $8^{\text {th }}$ edition AJCC staging for RB for both survival and eye globe salvage was evaluated by 8 ophthalmic oncology centers from 13 countries over 6 continents. They found that the overall eye globe salvage rate without EBRT was 52\%, and the cumulative 5- year survival and eye globe salvage estimates by clinical TNM categories were $100 \%$ and $96 \%$ for category cT1a, $98 \%$ and $88 \%$ for $\mathrm{cT} 1 \mathrm{~b}, 98 \%$ and $60 \%$ for cT2a, $96 \%$ and $57 \%$ for cT2b, $89 \%$ and $25 \%$ for cT3 tumors, respectively $[20,21]$. Based on that large and heterogenous $\mathrm{RB}$ patient population, the $8^{\text {th }}$ edition AJCC RB Staging System was found to be capable of predicting metastasis-related mortality as well as eye globe-salvage $[20,21]$. In our study we analyzed a homogenous group of RB patients who were treated by the same team in a single center, and similarly, we found that the eye salvage rate was $95 \%$ for $\mathrm{T} 1$ tumors (98\% for $\mathrm{T} 1 \mathrm{a}$ and $93 \%$ for $\mathrm{T} 1 \mathrm{~b}$ ), $68 \%$ for $\mathrm{T} 2$ tumors (90\% for $\mathrm{T} 2 \mathrm{a}$ and $66 \%$ for $\mathrm{T} 2 \mathrm{~b}$ ), and $12 \%$ for $\mathrm{T} 3$ tumors. Base on that, we looked for more accurate and detailed clinical features that may harbor higher predictive power for eye globe salvage and we incorporated that in our proposed modified staging system.

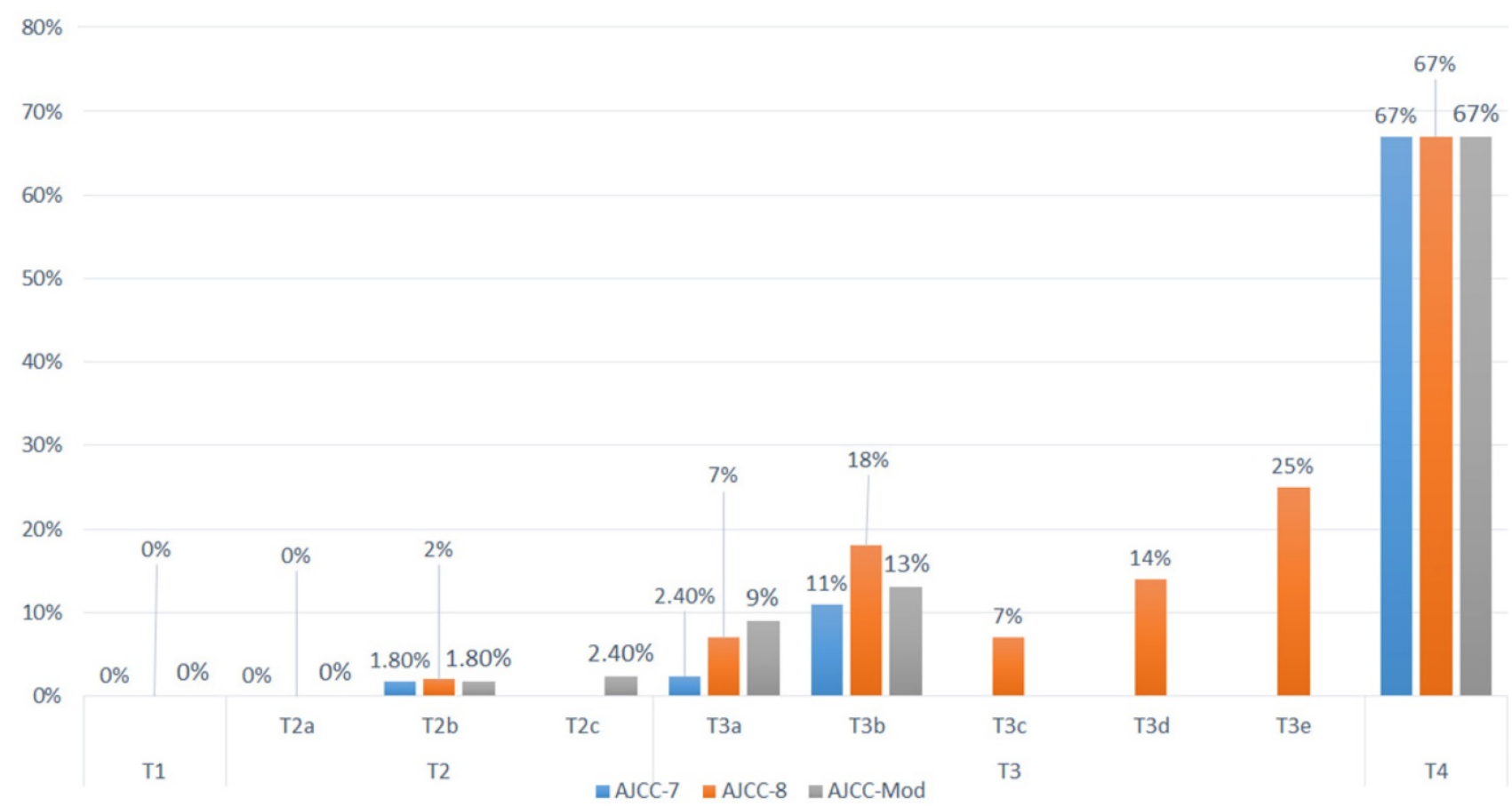

Figure 2. Metastatic Rates of Patients with Retinoblastoma. The metastatic rates are shown for tumors classified with the American Joint Committee on Cancer Staging Manual, $7^{\text {th }}$ edition and $8^{\text {th }}$ editions and our proposed cTNM staging system modifications. 
Table 6. Tumor cTNM Staging and Metastasis related Mortality (478 RB patients) a

\begin{tabular}{|c|c|c|c|}
\hline & Number (\%) & Metastasis & $\begin{array}{l}\text { Metastasis Related } \\
\text { Mortality }\end{array}$ \\
\hline $\begin{array}{l}\text { Total no. patients }{ }^{\mathrm{b}} \text { with } \\
\text { known stage }\end{array}$ & 362 patients & $17(5)$ & $16(4.4)$ \\
\hline Unknown stage & 116 patient & 4 & 4 \\
\hline \multicolumn{4}{|l|}{ AJCC-7 } \\
\hline T1 & $\underline{20(5.5)}$ & $\underline{0(0)}$ & $\underline{0(0)}$ \\
\hline $\mathrm{T} 1 \mathrm{a}$ & $5(1.4)$ & $0(0)$ & $0(0)$ \\
\hline $\mathrm{T} 1 \mathrm{~b}$ & $10(2.4)$ & $0(0)$ & $0(0)$ \\
\hline $\mathrm{T} 1 \mathrm{c}$ & $5(1.4)$ & $0(0)$ & $0(0)$ \\
\hline $\mathrm{T} 2$ & $179(50)$ & $\underline{3(1.7)}$ & $3(1.7)$ \\
\hline $\mathrm{T} 2 \mathrm{a}$ & $19(5)$ & $0(0)$ & $0(0)$ \\
\hline $\mathrm{T} 2 \mathrm{~b}$ & $160(44)$ & $3(1.8)$ & $3(1.8)$ \\
\hline T3 & $\underline{157(43)}$ & $\underline{10(6.4)}$ & $\underline{9(5.7)}$ \\
\hline T3a & $84(23)$ & $2(2.4)$ & $2(2.4)$ \\
\hline $\mathrm{T} 3 \mathrm{~b}$ & $73(20)$ & $8(11)$ & $7(9.5)$ \\
\hline $\mathrm{T} 4^{\mathrm{b}}$ & $\underline{6(1.6)}$ & $\underline{4(67 \%)}$ & $\underline{4(67 \%)}$ \\
\hline \multicolumn{4}{|l|}{ AJCC- 8} \\
\hline T1 & $\underline{15(4)}$ & $\underline{0(0)}$ & $\underline{0(0)}$ \\
\hline T1a & $5(1.4)$ & $\overline{0(0)}$ & $\overline{0(0)}$ \\
\hline $\mathrm{T} 1 \mathrm{~b}$ & $10(2.8)$ & $0(0)$ & $0(0)$ \\
\hline T2 & $268(74)$ & $\underline{5(2)}$ & $\underline{5(2)}$ \\
\hline $\mathrm{T} 2 \mathrm{a}$ & $5(1.4)$ & $0(0)$ & $0(0)$ \\
\hline $\mathrm{T} 2 \mathrm{~b}$ & $263(73)$ & $5(2)$ & $5(2)$ \\
\hline T3 & $73(20)$ & $8(11)$ & $7(9.5)$ \\
\hline Т3a & $23(3)$ & $2(7)$ & $2(7)$ \\
\hline $\mathrm{T} 3 \mathrm{~b}$ & $11(2)$ & $2(18)$ & $2(18)$ \\
\hline T3c & $28(4)$ & $2(7)$ & $1(4)$ \\
\hline T3d & $7(1)$ & $1(14)$ & $1(14)$ \\
\hline T3e & $4(1)$ & $1(25)$ & $1(25)$ \\
\hline $\mathrm{T} 4$ & $\underline{6(1.6)}$ & $\underline{4(67 \%)}$ & $\underline{4(67 \%)}$ \\
\hline $\mathrm{T} 4 \mathrm{a}$ & $5(<1)$ & 3 & 3 \\
\hline $\mathrm{T} 4 \mathrm{~b}$ & $1(<1)$ & 1 & 1 \\
\hline \multicolumn{4}{|c|}{ Proposed Modified cTNM Staging System } \\
\hline T1 & $20(5.5)$ & $0(0)$ & $0(0)$ \\
\hline T1a & $5(1.4)$ & $0(0)$ & $0(0)$ \\
\hline $\mathrm{T} 1 \mathrm{~b}$ & $10(2.8)$ & $0(0)$ & $0(0)$ \\
\hline T1c & $5(1.4)$ & $0(0)$ & $0(0)$ \\
\hline $\mathrm{T} 2$ & $263(73)$ & $5(2)$ & $5(2)$ \\
\hline $\mathrm{T} 2 \mathrm{a}$ & $19(5.3)$ & $0(0)$ & $0(0)$ \\
\hline $\mathrm{T} 2 \mathrm{~b}$ & $160(44)$ & $3(1.8)$ & $3(1.8)$ \\
\hline $\mathrm{T} 2 \mathrm{c}$ & $84(23)$ & $2(2.4)$ & $2(2.4)$ \\
\hline T3 & $73(20)$ & $8(11)$ & $7(9.5)$ \\
\hline $\mathrm{T} 2 \mathrm{a}$ & $35(5)$ & $3(9)$ & $2(6)$ \\
\hline $\mathrm{T} 2 \mathrm{~b}$ & $38(6)$ & $5(13)$ & $5(13)$ \\
\hline $\mathrm{T} 4$ & $6(1.4)$ & $4(67 \%)$ & $4(67 \%)$ \\
\hline Metastasis ${ }^{b}$ & $22 / 478(4.6)$ & & \\
\hline $\begin{array}{l}\text { Metastasis related } \\
\text { Mortality }\end{array}$ & $21(4.4 \%)$ & & \\
\hline \multicolumn{4}{|c|}{$\begin{array}{l}\text { Abbreviations: AJCC-7, American Joint Committee on Cancer Staging Manual, 7th } \\
\text { edition; AJCC-8, American Joint Committee on Cancer Staging Manual, 8th edition; } \\
\text { cTNM, clinical tumor/node/metastasis. }\end{array}$} \\
\hline \multicolumn{4}{|c|}{$\begin{array}{l}\text { a We correlated between the metastasis and the stage of the worst affected eye for } \\
\text { patients with bilateral disease. }\end{array}$} \\
\hline \multicolumn{4}{|c|}{$\begin{array}{l}\text { b We evaluated data from } 478 \text { patients, } 116 \text { patients had one eye enucleated before } \\
\text { referral to our center, therefore the stage of the worst eye wasn't known. }\end{array}$} \\
\hline
\end{tabular}

We divided cT2 tumors into $\mathrm{T} 2 \mathrm{a}$ to $\mathrm{T} 2 \mathrm{~b}$ subgroups according to the presence or absence of tumor seeds, which was supported by our finding that $90 \%$ of eyes without tumor seeding were salvaged. However, the cT2b subgroup encompassed a large group of heterogeneous tumors because this subgroup included all tumors with seeding, regardless of their location (subretinal vs vitreal) or severity (focal seeds vs massive seeds). The American
Joint Committee on Cancer Ophthalmic Oncology Task analyzed 592 eyes in group cT2b (AJCC-8) that had complete data for globe salvage analysis [5]. They found that the 5-year Kaplan-Meier cumulative globesalvage was $78 \%$ for eyes with focal seeding and $49 \%$ for eyes with diffuse seeding, and Cox proportional hazards regression analysis confirmed a higher local treatment failure risk with diffuse seeds as compared with focal seeds (hazard rate: 2.8; $p<0.001$ ) [5]. In the other hand there was insufficient evidence to prove or disprove an association between tumor seeds type and local treatment failure risk $(\mathrm{p}=0.06)$. Similarly, when we analyzed 368 T2b (AJCC-8) eyes that received conservative therapy, we found that tumors with massive seeds were more likely to fail treatment than those with focal seeds, as $74 \%$ of eyes with focal seeds in our series were salvaged, while only $46 \%$ of eyes with massive seeds were salvaged $(P=0.0005)$. Notably, in our series the type of seeding did not significantly affect the salvage rate $(P=0.161)$, although eyes with vitreous seeds were marginally less likely to be salvaged (61\%) than were those with subretinal seeds $(68 \%)$.

Although international RB staging systems can successfully predict the likelihood of eye salvage with intravenous chemotherapy $[6,7,8]$, the effect of tumor size on globe salvage prognosis remains controversial in the ocular oncology community. Two published versions of an international RB classification system are widely used: the International Intraocular Retinoblastoma Classification (IIRC) [4] and the Intraocular Classification for Retinoblastoma (ICRB) [5]. Although both systems are very similar, they contain subtle differences, most notably in their classification of group $\mathrm{E}$ eyes. The ICRB considers large tumors (ie, $>50 \%$ of the globe) as group $\mathrm{E}$, whereas these tumors would be assigned as group B, $\mathrm{C}$, or D by the IIRC. Because of these discrepancies, the international staging system is inconsistent, which is also the case for AJCC-7 and AJCC-8. AJCC-7 upstages tumors from $\mathrm{T} 2$ to $\mathrm{T} 3 \mathrm{a}$ if the tumors fill more than two-thirds of the globe and to T3b if the tumors are associated with destroyed eye structures, such as neovascular or angle closure glaucoma, tumor extension into the anterior segment, hyphema, vitreous hemorrhage, or orbital cellulites. Because AJCC-8 excludes tumor size from cT3 grouping, group cT3 tumors have only the features of group $\mathrm{E}$ tumors, according to the IIRC [4]. To resolve this issue, we propose modifications on $\mathrm{T} 2$ grouping in AJCC-8, and we considered specific subgroup for tumors occupying more than two-thirds of the globe (cT2c). The likelihood of eye salvage for these large tumors was $25 \%$, which is better than the unsalvageable cT3 (IIRC group E) tumors but still 
worse than small tumors with focal or massive seeds (T2a and $\mathrm{T} 2 \mathrm{~b}$ ).

We believe it is important to consider IIRC group E tumors (stage CT3 in AJCC-8) as a solitary group because although they comprise intraocular tumors, these tumors are at higher risk of treatment failure than are T1 and T2 tumors. These tumors harbor high-risk histopathologic features that predispose them to an increased risk of systemic metastasis $[15,22,23,24,25]$.

In our proposal, we classified cT3 group into 2 subgroups; cT3a are eyes with features secondary to ocular ischemia (including neovascular glaucoma, buphthalmous, hyphaema, and vitreous hemorrhage), and cT3b are eyes with tumor invasion into vital structures that may cause metastasis (including ciliary body and anterior chamber invasion, phthisis, and orbital cellulitis).

cT3a eyes are expected to have low potential for eye and vision salvage because of ischemia, but relatively lower chance of metastasis as they do not invade vital structures and will not show high risk pathological features in comparison to cT3b eyes [16, 17]. The low number of patients in group cT3 in our study did not help us to prove our theory about dividing cT3 eyes into 2 subgroups. In the literature, there is a significant disparity regarding the risk of metastasis associated with high-risk clinical features. Chantada et al and Kim et al suggested that glaucoma is significantly associated with high-risk pathology. In these reports, authors compared the chance of HRPF between eyes with glaucoma and eyes without glaucoma, but they did not compare that with eyes with anterior segment invasion and/or phthisis [26, 27]. Similarly, Kashyap et al concluded that vitreous hemorrhage, hyphema, staphyloma, and orbital cellulitis were predictors of high-risk pathology, but again in comparison with less advanced tumors [28]. Furthermore, eyes with hyphaema will be more difficult to assess for anterior chamber and ciliary body invasion clinically unless they used specific imaging studies as ultrasound biomicroscopy. Based on that, there is no evidence until now about which clinical signs of advanced intraocular RB are associated with worse prognosis than others. Hopefully bigger international studies may be done in the future to validate dividing cT3 into 2 subgroups or more that show difference in survival, and that be with or against our theory.

\section{Strengths and Limitations}

The same clinical team consistently managed all of the tumors in our analysis, and the sample size and tumor diversity in our cohort yielded statistical significance, strengthening our study. The retrospective nature, analysis of only intraocular $\mathrm{RB}$ tumors, and omission of survival prognosis were limitations in our study. Furthermore, our results are single-center data that may be not directly comparable to that derived from patients around the world.

\section{Conclusions}

The clinical staging criteria used in the AJCC-7 and AJCC-8 TNM staging systems can predict the likelihood of globe salvage with conservative treatment. Tumors with massive seeding and large tumors are best considered as a more advanced stage. Accordingly, we propose modifications that outperform the current cTNM staging system and may resolve the discrepancies in previously published RB classification systems.

\section{Supplementary Material}

Supplementary tables.

https://www.jcancer.org/v13p1336s1.pdf

\section{Acknowledgements}

The authors declare no conflicts of interest. We acknowledge Nisha Badders, PhD, ELS (Scientific Editor, St. Jude Children's Research Hospital) for the comprehensive language and scientific editing of the manuscript. We also acknowledge Ayat Taqash (King Hussein Cancer Center, Amman, Jordan) for providing assistance with the statistical analysis in this study.

\section{Funding}

This research was supported in part by King Hussein Cancer Center and The Medical University of Lublin.

\section{Author Contributions}

Conceptualization, Y.A.Y., I.Q. and M.A.; methodology, M.A.,Y.A.Y, I.A. and M.D.T.; software, D.A., I.J., I.S. and I.A.; validation, Y.A.Y., D.A., I.A., I.Q. and M.D.T.; formal analysis, S.Z., R.R., R.N. and M.M.; investigation, I.Q., Y.A.Y., M.M., R.N. and M.M.; resources, Y.A.Y., M.D.T. and R.N.; data curation, Y.A.Y., I.Q. and M.Me.; writing-original draft preparation, Y.A.Y., I.Q., M.D.T., M.M., S.Z., R.R. and R.N.; writing-review and editing, all authors; visualization, I.Q., I.A. and M.A.; supervision, D.A., R.R. and M.D.T.; project administration, Y.A.Y., M.M., I.J., I.S., I.A., M.A. and M.D.T.; funding acquisition M.D.T. and Y.A.Y. All authors have read and agreed to the published version of the manuscript. 


\section{Institutional Review Board Statement}

The study was conducted according to the guidelines of the Declaration of Helsinki, and approved by the Institutional Review Board of King Hussein Cancer Center (20khcc08, March 2020).

Informed Consent Statement: Patient consent was waived due to the retrospective study design.

\section{Competing Interests}

The authors have declared that no competing interest exists.

\section{References}

1. Dimaras H, Kimani K, Dimba EA, Gronsdahl P, White A, Chan HS, Gallie BL. Retinoblastoma. Lancet. 2012; 379(9824):1436-46.

2. Kivelä T. The epidemiological challenge of the most frequent eye cancer: retinoblastoma, an issue of birth and death. Br J Ophthalmol. 2009;93(9):1129-31.

3. Yousef YA, Al-Nawaiseh I, Mehyar M, Sultan I, Al-Hussaini M, Jaradat I, et al. How Telemedicine and Centralized Care Changed the Natural History of Retinoblastoma in a Developing Country: Analysis of 478 Patients. Ophthalmology. 2021;128(1):130-7.

4. Dimaras H, Corson TW, Cobrinik D, White A, Zhao J, Munier FL, et al. Retinoblastoma. Nat Rev Dis Primers. 2015;1:15021.

5. Tomar AS, Finger PT, Gallie B, Kivelä T, Mallipatna A, Zhang C, et al. Retinoblastoma seeds: impact on American Joint Committee on Cancer clinical staging. Br J Ophthalmol. 2021; [Epub ahead of print]

6. Linn Murphree A. Intraocular retinoblastoma: The case for a new group classification. Ophthalmol Clin North Am. 2005;18:41-53.

7. Shields CL, Mashayekhi A, Au AK, Czyz C, Leahey A, Meadows AT, et al. The international classification of retinoblastoma predicts chemoreduction success. Ophthalmology. 2006;113:2276-80.

8. Munier FL, Beck-Popovic M, Chantada GL, Cobrinik D, Kivelä TT, Lohmann $\mathrm{D}$, et al. Conservative management of retinoblastoma: Challenging orthodoxy without compromising the state of metastatic grace. Alive, with good vision and no comorbidity. Prog Retin Eye Res. 2019;73:100764.

9. Shields CL, Bas Z, Tadepalli S, Dalvin LA, Rao R, Schwendeman R, et al. Long-term (20-year) real-world outcomes of intravenous chemotherapy (chemoreduction) for retinoblastoma in 964 eyes of 554 patients at a single centre. Br J Ophthalmol. 2020;104(11):1548-55.

10. Kim JW, Shah SN, Green S, O'Fee J, Tamrazi B, Berry JL. Tumour size criteria for Group $D$ and $E$ eyes in the International Classification System for Retinoblastoma: effects on rates of globe salvage and high-risk histopathologic features. Acta Ophthalmol. 2020; 98(1):e121-e125.

11. Abramson DH, Sheilds CL, Munier FL, Chantada GL. Treatment of retinoblastoma in 2015: agreement and disagreement. JAMA Opthalmol. 2015; 133(11):1341-47

12. [No authors listed] Canadian Retinoblastoma Society. National Retinoblastoma Strategy Canadian Guidelines for Care: Stratégie thérapeutique du rétinoblastome guide clinique canadien. Can J Ophthalmol. 2009;44 (Suppl 2:S1-88.

13. Yousef YA, Soliman SE, Astudillo PPP, Durairaj P, Dimaras H, Chan HSL, et al. Intra-arterial chemotherapy for retinoblastoma: a systematic review. JAMA Ophthalmol. 2016;134(5):584-591.

14. Abramson DH, Fabius AW, Issa R, Francis JH, Marr BP, Dunkel IJ, Gobin YP. Advanced Unilateral Retinoblastoma: The Impact of Ophthalmic Artery Chemosurgery on Enucleation Rate and Patient Survival at MSKCC. PLoS One. $2015 ; 10(12): \mathrm{e} 0145436$

15. In: Edge SD, Byrd DR, Carducci MA, Compton CC, eds. Chapter 44: Retinoblastoma. AJCC Cancer Staging Manual. 7th ed. New York, NY: Springer; 2009.

16. Mallipatna AC, Gallie BL, Chévez-Barrios $P$, et al. Retinoblastoma. In: Amin MB, Edge SB, Greene FL, Byrd DR, Brookland RK, Washington MK, et al., editors. AJCC Cancer Staging Manual. 8th ed. New York: Springer; 2017. pp. 819-31.

17. Yousef YA, Al-Hussaini M, Mehyar M, Yousef YA, Al-Hussaini M, Mehyar M, Sultan I, Jaradat I, AlRawashdeh $\mathrm{K}$, et al. The predictive value of TNM classification, The International Classification, and Reese- Ellsworth Staging of retinoblastoma for the likelihood of high risk pathologic features. Retina. 2015;35(9):1883-9.

18. Wilson MW, Qaddoumi I, Billups C, Haik BG, Rodriguez-Galindo C. A clinicopathological correlation of 67 eyes primarily enucleated for advanced intraocular retinoblastoma. Br J Ophthalmol. 2011;95(4):553-8.

19. Harrell FE, Jr., Lee KL, Mark DB. Multivariable prognostic models: issues in developing models, evaluating assumptions and adequacy, and measuring and reducing errors. Stat Med. 1996;15:361-87.

20. Fabian ID, Reddy A, Sagoo MS. Classification and staging of retinoblastoma. Community Eye Health. 2018; 31(101):11-13.
21. Chantada GL, Sampor C, Bosaleh A, Solernou V, Fandiño A, de Dávila MT. Comparison of staging systems for extraocular retinoblastoma: analysis of 533 patients. JAMA Ophthalmol. 2013;131(9):1127-34.

22. Tomar AS, Finger PT, Gallie B, Mallipatna A, Kivelä TT, Zhang C, et al. A Multicenter, International Collaborative Study for American Joint Committee on Cancer Staging of Retinoblastoma: Part I: Metastasis-Associated Mortality. Ophthalmology. 2020;127(12):1719-32.

23. Tomar AS, Finger PT, Gallie B, Mallipatna A, Kivelä TT, Zhang C, et al. A Multicenter, International Collaborative Study for American Joint Committee on Cancer Staging of Retinoblastoma: Part II: Treatment Success and Globe Salvage. Ophthalmology. 2020;127(12):1733-46.

24. Chantada GL, Dunkel IJ, de Davila MT, Abramson DH. Retinoblastoma patients with high risk ocular pathological features: who needs adjuvant therapy? Br J Ophthalmol. 2004; 88(8):1069-73.

25. Eagle RC, Jr. High-risk features and tumor differentiation in retinoblastoma: a retrospective histopathologic study. Arch Pathol Lab Med. 2009;133(8):1203-9.

26. Chantada GL, Gonzalez A, Fandino A, de Davila MT, Demirdjian G, Scopinaro M, Abramson D. Some clinical findings at presentation can predict high-risk pathology features in unilateral retinoblastoma. J Pediatr Hematol Oncol. 2009;31(5):325-9.

27. Kim ME, Shah S, Zolfaghari E, Jubran R, Reid MW, Kim JW, Berry JL. . An Intraocular Pressure Predictive of High-risk Histopathologic Features in Group E Retinoblastoma Eyes. Int Ophthalmol Clin. 2019;59(2):77-86.

28. Kashyap S, Meel R, Pushker N, Sen S, Bakhshi S, Sreenivas V, Sethi S, et al. Clinical predictors of high risk histopathology in retinoblastoma. Pediatr Blood Cancer. 2012;58(3):356-61 